\title{
Evaluation of Blunt Abdominal Trauma by Helical Spiral CT Scan - A One Year Study at a Tertiary Care Hospital
}

\author{
Ritesh Kondeti ${ }^{1}$, Gopinath Loganathan ${ }^{2}$ \\ ${ }^{1}$ Associate Professor, ${ }^{2}$ Assistant Professor, Department of Radiology, Pondicherry Institute of Medical Sciences, Pondicherry, \\ India
}

Corresponding author: Dr. Gopinath Loganathan, Assistant Professor, Department of Radiology, Pondicherry Institute of Medical Sciences Kalapet Pondicherry 605014, India

DOI: 10.21276/ijcmsr.2018.3.3.25

How to cite this article: Ritesh Kondeti, Gopinath Loganathan. Evaluation of blunt abdominal trauma by helical spiral CT scan- a one year study at a tertiary care hospital. International Journal of Contemporary Medicine Surgery and Radiology. 2018;3(3):C112-C115.

\section{A B S T R A C T}

Introduction: Trauma is the leading cause of death in modern era of developing world and accounts for 10-15 million deaths globally. Blunt abdominal trauma (BAT) is a medical or surgical emergency and is associated with significant morbidity and mortality despite advances in diagnosis, management and intervention. The present study aimed to detect the findings in helical spiral CT scan in cases of BAT and also to assess the outcome with relation to time of presentation.

Material and Methods: A one year prospective study was conducted by department of radiology in association with emergency medicine. All cases of blunt abdominal trauma who fulfilled the inclusion criteria was performed helical spiral CT scan and findings were noted and reported. CT Cystography was performed in cases of pelvic fractures or history of Hematuria or blood at urethral meatus.

Results: 85 cases were included with 56 males and 29 females. 31-40 years was the most common age group with mean age of $31.20 \pm 1.6$ years. Spleen was the major organ injured followed by liver and kidney in our study. Grade-I splenic injury was common and in liver also grade-I was common. Hemoperitoneum was observed in $51.21 \%$ cases and the fluid collection was moderate in $71.42 \%$ cases. Retroperitoneal haemorrhage was observed in $34.24 \%$ of cases.

Discussion: In conjunction with clinical examination and monitoring CT is an accurate, safe and was reliable in initial evaluation cases of blunt abdominal trauma. With the accuracy of CT in cases of BAT the rate of negative laporatomy will be reduced by avoiding surgical intervention in cases which can be managed conservatively.

Key words: Blunt Abdominal Injury, Hemoperitoneum, Retroperitoneal Haemorrhage

\section{INTRODUCTION}

Trauma is the leading cause of death in modern era of developing world and accounts for 10-15 million deaths globally. Over the world trauma is the $7^{\text {th }}$ cause of mortality and abdomen is the third affected site in the cases of polytrauma preceded by head and chest. $25 \%$ of cases of trauma of abdomen require surgery and $85 \%$ of abdominal injuries are blunt in nature. ${ }^{1}$ Blunt abdominal trauma (BAT) is a medical or surgical emergency and is associated with significant morbidity and mortality despite advances in diagnosis, management and intervention. Clinical examination alone is not sufficient or inadequate in arriving at a diagnosis as most of the cases present with altered mental status and distracting other injuries. Most of the cases of BAT have spleen or liver as most common injured organs with associated damage to the bowel and mesentery. ${ }^{2}$ Ultra sonogram of the abdomen is useful investigation in detection of major injuries. However initial resuscitation along with focussed assessment with sonography in trauma (FAST) along with Computed Tomography of abdomen is a useful diagnostic modality in accessing minor injuries to the abdominal organs with associated fluid collections in the peritoneal cavity. ${ }^{3}$ The introduction of helical CT in 1980s has improved the detection and classification of Blunt abdominal injury. Further developments in the CT technology, introduction and refinement of multidetector scanners have fully expanded the versatility of CT diagnostic modality in cases of BAT and polytrauma cases. Advantages of a multidetector CT scan are higher spatial resolution, faster image acquisition and reconstruction, and improved patient safety. Some authors recommend MDCT imaging survey as a standard diagnostic tool during early resuscitation survey in cases of polytrauma. A MDCT scan of the chest or abdomen may cause a change in 30\% of cases in management and treatment. Now most of the studies suggest that MDCT scanning with intravenous contrast is the gold standard diagnostic modality in cases of BAT who are hemodynamically stable. ${ }^{4}$ The time of presentation plays a key role has a large impact on the outcome of the patients. The present study aimed to detect the findings in helical spiral CT scan in cases of BAT and also to assess the outcome with relation to time of presentation. 


\section{MATERIAL AND METHODS}

A one year prospective study was conducted at a tertiary care center by the department of radiology from January 2106 to December 2016. The study protocol was presented to the institutional ethical committee and all the guidelines of the committee were followed after approval. The details of the study were explained to all the cases in the study or relatives or Guardians in case the patient was unconscious. Informed and written consent was obtained from all the cases in the study. Patient's referred to the radiology department from Emergency or causality with blunt injury to abdomen within 24 hours was enrolled as cases in the study. Initial evaluation of the cases was done by ultrasonogram (USG) and Helical CT of abdomen was carried out in cases where USG findings were inconclusive or for evaluation of integrity of hollow viscera or for better characterization of injuries made out on USG.

\section{CT Protocols}

CT scan of abdomen was performed with Hi speed GE Helical Scanner with $30 \mathrm{~s}$ spiral length and $1 \mathrm{~s}$ time. $15 \mathrm{ml}$ of oral contrast Gastroscan mixed in one litre of normal saline and administered either orally in stable cases or through Ryle's tube over a period of 45-60 minutes. With the help of pressure injector about $100 \mathrm{ml}$ of non-ionic contrast, Iopamiro was administered intravenously at a flow rate of $1.5 \mathrm{ml}$ / second. Ryle's tube was withdrawn after adequate length so as to avoid streak artefacts. Volume scans of abdomen and pelvis were usually obtained with $7 \mathrm{~mm}$ collimation, a pitch of 1.5 and 50s scan delay. Region of interest was from domes of diaphgram to inferior edge of Ischia. Thinner sections and delayed scans were studied as and when required. Window settings were used depending on the findings. CT Cystography was performed in cases of pelvic fractures or history of Hematuria or blood at urethral meatus by infusion of about $500 \mathrm{ml}$ of diluted contrast through foley's catheter by gravity method. Triple contrast were given in cases where there was hematochezia or flank injury in which case there are more chances of injury to large bowel.

The CT findings were correlated with the final outcome as indicated by duration of hospital stay or if managed conservatively and with laparotomy findings on case to case basis.

\section{STATISTICAL ANALYSIS}

Descriptive statistics like mean and percentages were used for the analysis. Microsoft office 2007 was used for the analysis.

\section{RESULTS}

A total of 85 cases of blunt abdominal trauma were included in the study and written consent was obtained from all the cases in the study. Males were predominant in the study with $65.9 \%$ and females with $34.1 \%$. The mean age of the study group was $31.20 \pm 1.6$ years. The mean age of the males in the study was $26.12 \pm 2.8$ years and females was $29.21 \pm 1.6$ years. Majority of the cases of BAT was observed in the age group of 31-40 years with $30.6 \%$ followed by $21-30$ years with $23.5 \%$ and least common in 51-60 years with $3.5 \%$. Road traffic accidents $(42.35 \%)$ and fall from a height $(24.71 \%)$

\begin{tabular}{|l|l|c|c|}
\hline S No & Organ & No of cases & $\%$ \\
\hline 1 & Spleen & 32 & 37.65 \\
\hline 2 & Liver & 24 & 28.24 \\
\hline 3 & kidneys & 10 & 11.76 \\
\hline 4 & Bowel & 14 & 16.47 \\
\hline 5 & Adrenal glands & 4 & 4.71 \\
\hline 6 & Pancreas & 6 & 7.06 \\
\hline 7 & mesentery & 11 & 12.94 \\
\hline 8 & Urinary Bladder & 6 & 7.06 \\
\hline \multicolumn{2}{|c|}{ Table-1: Distribution of Organ injury among cases in the study } \\
\hline
\end{tabular}

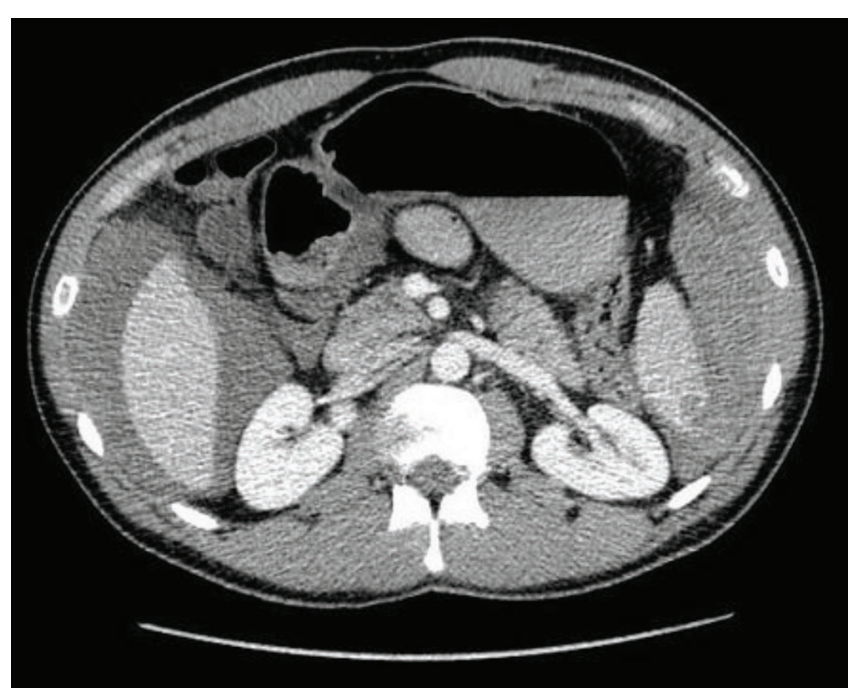

Figure-1: CT image showing hyperdense areas around the spleen, secondary to splenic injury

accounted for more than $50 \%$ of cases in the study. Other causes were collision injury, domestic violence, fall of heavy objects etc. However in the present study associated injuries to the chest $(33.4 \%)$, head $(26.2 \%)$ and other solid organs (25.1\%) were observed. Other associated injuries noted were fracture of the rib, contusion of the lung, hemothorax, and pneumothorax. Splenic injury was the most common observation in our study with $37.65 \%$. Splenic injury was associated with injury to liver (12.63\%), kidneys (8.12\%) and Pancreas (4.1\%). Splenic injury was classified based on Mirvis classification into Grade-I to IV. The major findings observed on CT scan evaluation were splenic contusion and hematoma (Grade-I - 21/32) followed by Splenic laceration $<3 \mathrm{~cm}$ (Grade-II, 6/32), Splenic laceration $>3 \mathrm{~cm}$ (GradeIII, 4/32) and devascularized spleen (Grade- IV, 1/32). [TABLE-1]

Liver was the second common organ injured in the cases of the study with $28.24 \%$. The injury was classified based on the Organ injury scale of the liver to Grade-I to Grade-VI. Majority of the cases were with Grade-I injury (Sub capsular hematoma with $<10 \%$ surface area and capsular tear with $<1 \mathrm{~cm}$ depth) with $14 / 24$ cases followed by Grade-II (6/24), Grade-III (2/24) and Grade-IV (2/24).

Renal injury was observed in 10 cases of the study and was graded from I to IV. Majority were present in Grade-I with 8/10 and 1 each with Grade-II and Grade-III. Other injuries observed in the study were injury to the bowel, adrenal glands, pancreas, mesentery and urinary bladder. 
In cases of hemoperitoneum, the amount of fluid was quantified as minimal, moderate or marked based on the observations on consecutive CT scans. Intraperitoneal fluid estimation by volumetric analysis showed minimal fluid to be less than $50 \mathrm{ml}$, moderate fluid collections to be less than $150 \mathrm{ml}$ and large fluid collections to be greater than $150 \mathrm{ml}$. The cumulative amounts of fluid was rated as small $(+1)$, intermediate $(+2)$ and large $(+3)$ depending on the sum of amount of fluid in the individual intra peritoneal locations. Hemoperitoneum was observed in $51.21 \%$ cases with minimal in $9.52 \%$ cases, moderate in $71.42 \%$ cases and marked in $19.04 \%$ of cases in the study. Free fluid was seen in $36.58 \%$ of cases. [FIG-1] Retroperitoneal haemorrhage was observed in $34.24 \%$ of cases with central in $14.28 \%$, perinephric in $56.12 \%$ and pelvic in $28.54 \%$ of cases. 'Sentinel Clot' was observed in $9.5 \%$ of cases. Intravenous contrast extravasation was noticed in $7.31 \%$ of cases of which 2 cases were of splenic vasculature and 1 from renal vessels. Oral contrast extravasation was seen in 2 cases of which one from duodenum and other from ileum.

Of the 85 cases included in the study, 32 cases (37.65\%) had splenic injury, 24 (28.24\%) had hepatic injury, 14(16.47\%) had injury to bowel, $11(12.94 \%)$ cases with injury to mesentery,10 cases (11.76\%) with renal injury, 6 cases each $(7.06 \%)$ with bladder and pancreas injury and 4 cases (4.71\%) with injury to adrenals. Combined organ injuries were observed in 24 cases (28.24\%).

Mesenteric injuries were made out as haziness of mesentery, discontinuity of mesenteric vascular arcade and fat stranding adjacent to site of injury to mesentery. Other miscellaneous injuries made out were psoas hematoma, anterior abdominal wall and pelvic hematomas.

\section{DISCUSSION}

Several studies have reported the inaccuracies in diagnosing the cases of blunt abdominal trauma with only clinical examination. Hence the present study highlights the supportive role of computed tomography in accurate diagnosis and management decisions in outcome of the patient. As highlighted in many studies our study also had a male preponderance of cases which is explained the fact that men are more exposed to work and traffic related activities. Middle aged group between 31-40 years were most common group in our study and also the same pattern was observed in various studies universally as this is the age group highly involved in work and other active strenuous injury related acts like violence and fallouts. Most studies stated that CT plays an crucial role in identification of internal injuries in blunt abdominal trauma including organ and bowel and mesentery. Role of CT in Identification of fluid collection in the peritoneum (hemoperitoneum) is always a debate and many studies suggest that identification depends on the amount of fluid collected i.e, mild, moderate and marked.5, Splenic injury was the most common injury in our study with $37.65 \%$ which is similar to the reports of Miller et al who reported a slightly higher incidence in his study with $42 \%$ but was significantly low in the study of Dolich et al who reported an incidence of only $16 \%$ in his study. However the variation may be due to the type of injury associated with the study and type of imaging modality and type of CT used in assessing the injury. Splenic contusion and hematoma was the most common finding in our study as observed in many other studies. ${ }^{7,8}$

Liver was the second major organ injured in our study which is similar to the observation of Nolan et al who reported the incidence as 155 which is on par with the findings of our study. ${ }^{9}$ Majority of the studies conducted related to blunt abdominal injury states that injury to the liver is associated with other organ injuries like chest, skull in case of collision injuries and fallouts. In case of injury to liver Grade-I was the commonest finding (Sub capsular hematoma with $<10 \%$ surface area and capsular tear with $<1 \mathrm{~cm}$ depth) and is similar to the finding of $\mathrm{Vu} \mathrm{M}$ who reported $12 \%$ in his cases of liver injury. ${ }^{10}$ Renal injury was the third most common in the studywith only 10 cases and was similar to the findings of Dayal $\mathrm{M}$ who reported the same in his study, however few studies reported that renal injury was the second most common injury after spleen and is commonly associated with injury to injury to adrenals, pancreas and mesentery. ${ }^{11}$ Renal injury was graded and majority of the cases in our study were of grade-I and study of Marco GG has reported the same with an incidence of $28 \%$ of grade-I renal injury in his study. $^{12}$

Hemoperitoneum was observed in $51 \%$ of cases in our study which was significantly higher when compared to the findings of Bhagavan $\mathrm{S}$ who reported an incidence of only $28 \%$ in his study. However the variation may be explained by the reason that type of imaging technique used was FAST by Bhagavan whereas Helical Ct was used as imaging technique in our study which had high sensitivity and specificity than FAST. Other studies that used Helical CT for blunt abdominal injury reported a similar incidence of Hemoperitoneum in their studies. ${ }^{13}$ Hemoperitoneum as per the grading in majority of the cases was moderate and in majority of cases with fluid collection, perforation of hollow viscus was noticed. Similar findings were reported from the study of Federle M et al. ${ }^{14}$ Retroperitoneal haemorrhage was observed in $34.24 \%$ of cases in our study which was also reported in the study of Scaglione $\mathrm{M}$ et al. ${ }^{15}$ The position of retroperitoneal haemorrhage was pelvic in majority of the cases.

\section{CONCLUSION}

To conclude, in conjunction with clinical examination and monitoring CT is an accurate, safe and was reliable in initial evaluation cases of blunt abdominal trauma. The sensitivity as well as specificity was higher in solid organ injuries. However the results exhibited lower sensitivity and specificity in bowel, mesentery and vascular injuries. With the accuracy of CT in cases of BAT the rate of negative laporatomy will be reduced by avoiding surgical intervention in cases which can be managed conservatively.

\section{REFERENCES}

1. Mehta N, Babu S, Venugopal K. An Experience with Blunt Abdominal Trauma: Evaluation, Management and Outcome. Clinics and Practice. 2014;4(2):599.

2. Sauaia A, Moore FA, Moore EE, Moser KS, Brennan R, 
Read RA, Pons PT: Epidemiology of trauma deaths: a reassessment. J Trauma 1995;38(3):185-193.

3. Deunk J, Brink M, Dekker HM, Kool DR, Blickman JG, van Vugt AB,Edwards MJ: Routine versus selective computed tomography of the abdomen, pelvis, and lumbar spine in blunt trauma: a prospective evaluation. J Trauma 2009;66(4):1108-1117.

4. Van der Vlies CH, Olthof DC, Gaakeer M, Ponsen KJ, van Delden OM, Goslings JC. Changing patterns in diagnostic strategies and the treatment of blunt injury to solid abdominal organs. International Journal of Emergency Medicine. 2011;4(6):47.

5. Aseervatham R, Muller M: Blunt trauma to the spleen. Aust NZ J Surg 2000;70(2):333-337.

6. Killeen KL, Shanmuganathan K, Poletti PA, Cooper C, Mirvis SE. Helical computed tomography of bowel and mesenteric injuries. J Trauma 2001;51(4):26-36.

7. Miller LA, Shanmuganathan K. Multidetector CT evaluation of abdominal trauma. Radiol Clin North Am 2005;43(5):1079-95.

8. Dolich MO, McKenney MG, Varela JE, Compton RP, McKenney KL,Cohn SM. 2,576 ultrasounds for blunt abdominal trauma. J Trauma 2001;50(3):108-12.

9. Nolan B, Gabram S, Schwartz R, et al. Mesenteric injury from blunt abdominal trauma. Am Surg 1995; 61(6):501-506.

10. $\mathrm{Vu} \mathrm{M}$, Anderson SW, Shah N, et al. CT of blunt abdominal and pelvic vascular injury. Emerg Radiol 2010;17(1):21-9.

11. Dayal M, Gamanagatti S, Kumar A. Imaging in renal trauma. World J. Radiol 2013;5(8):275-84.

12. Marco GG, Diego S, GiulioA, et al. Screening US and CT for blunt abdominal trauma: a retrospective study. Eur J Radiol 2005; 56(1): 97-101.

13. Bhagvan S, Turai M, Holden A, Ng A, Civil I. Predicting hollow viscus injury in blunt abdominal trauma with computed tomography. World J Surg 2013;37(3):123-6.

14. Federle M, Jeffery R. Hemoperitoneum studied by computed tomography. Radiology 1983;148(6):187192.

15. Scaglione M, de Lutio di Castelguidone E, Scialpi M, et al: Blunt trauma to the gastrointestinal tract and mesentery: is there a role for helical CT in the decisionmaking process? Eur J Radiol 2004;50(2):67-73.

Source of Support: Nil; Conflict of Interest: None

Submitted: 15-08-2018; Accepted: 26-08-2018; Published online: 31-08-2018 